\title{
A longitudinal study of the effects of ART on plasma chemokine levels in Malaysian HIV patients
}

\author{
Constance S.N. Chew ${ }^{\mathrm{a}}$, Catherine L. Cherry ${ }^{\mathrm{b}, \mathrm{c}, \mathrm{d}}$, Adeeba Kamarulzaman ${ }^{\mathrm{e}, \mathrm{f}}$, Tan Hong Yien ${ }^{\mathrm{e}, \mathrm{f}}$, \\ Zayd Aghafar ${ }^{\mathrm{a}}$ and Patricia Price ${ }^{\mathrm{a}, *}$

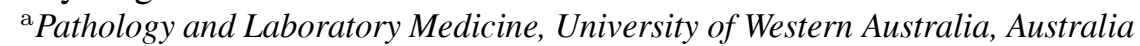 \\ ${ }^{\mathrm{b}}$ Centre for Virology, Burnet Institute, Melbourne, Australia \\ ${ }^{\mathrm{c}}$ Infectious Diseases Unit, Alfred Hospital, Melbourne, Australia \\ ${ }^{\mathrm{d}}$ Department of Medicine, Monash University, Melbourne, Australia \\ ${ }^{\mathrm{e}}$ Centre of Excellence for Research in AIDS (CERiA), Faculty of Medicine, University of Malaya, Kuala Lumpur, \\ Malaysia \\ ${ }^{\mathrm{f}}$ University Malaya Medical Centre, Kuala Lumpur, Malaysia
}

\begin{abstract}
Objectives: Chemokines influence the migration of leukocytes to secondary lymphoid tissue and sites of inflammation. In HIV patients, they are implicated in inflammatory complications of antiretroviral therapy (ART), notably Immune Reconstitution Disease (IRD) and Sensory Neuropathy (SN). However most chemokines have not been monitored as patients begin ART or correlated with IRD and SN.

Methods: Plasma chemokine levels were assessed longitudinally using commercial ELISAs in 69 patients treated in Kuala Lumpur, Malaysia. Plasma was available at baseline and after 6, 12, 24 and 48 weeks on ART. Chemokine genotypes were assessed using allele-specific fluorescent probes. IRD were diagnosed in 15 patients. 30 patients were screened for SN using the ACTG BPNS tool after six months on ART. SN was detected in 8 patients.

Results: Plasma CXCL10 levels decreased on ART compared to baseline ( $p=0.002-0.0001)$, but remain higher than healthy controls $(p \leqslant 0.0001)$. The decline was clearer in patients without IRD. CCL5 levels rose on ART but remained similar to controls. CCL2 levels were higher in patients than controls after week 12. Plasma chemokine levels were not affected by CD4+ T-cell counts or any genotypes tested. Several patients with SN displayed higher CCL5 levels throughout therapy compared to patients without neuropathy. Levels of other chemokines and chemokine genotypes were not associated with SN.

Conclusions: Chemokines are differentially affected by ART. CXCL10 and CCL5 may influence IRD and CCL5 warrants further investigation for an effect in SN. These trends are not influenced by chemokine genotypes investigated here.
\end{abstract}

Keywords: HIV sensory neuropathy, nucleoside analogue reverse transcriptase inhibitor sensory neuropathy, CCL5, CXCL10, Immune Reconstitution Disease

\section{Introduction}

Antiretroviral therapy (ART) has improved life expectancy in HIV-infected individuals by suppressing viral replication and reducing opportunistic infections.

* Address for correspondence: Professor Patricia Price, School of Pathology and Laboratory Medicine, University of Western Australia, Nedlands, 6009, Australia. Tel.: +61 6189224 0378; Fax: +61 6189224 0204; E-mail: patricia.price@uwa.edu.au.
However, several adverse effects compromise both patient health and adherence to therapy. These include Sensory Neuropathy (SN) [1] and Immune Reconstitution Disease (IRD) [2,3]. Both are characterized by dysregulated host inflammatory responses $[4,5]$. Circulating chemokines may be a component of functional immune reconstitution or may mediate SN or IRD.

Chemokines are critical for inflammation and the induction of adaptive immune responses, but the effects of ART on chemokines are not well characterized, and 
very few studies assess plasma chemokine levels in longitudinal sample sets. Increased levels of CCL5 were evident from 4 weeks on ART in two studies [6, 7]. CCL5 production by cultured CD4+ and CD8+ Tcells was associated with maintenance of undetectable plasma HIV RNA for 1 year [8]. Plasma CXCL10 levels decreased after 24 weeks of ART in patients from the USA and after 1 year on ART in Cambodian patients $[9,10]$. Persistently high CXCL10 levels were also associated with immunological treatment failure following ART [11]. However other chemokines have not been monitored over time in patients initiating ART.

Up to $45 \%$ of HIV-1 infected patients experience an IRD when numbers of memory CD4+ T-cells are rising on ART [3]. This parallels the restoration of immune responses against pre-existing opportunistic infections. The immune response may be immunopathological rather than protective (reviewed by Stone et al. [12]). Pathogens commonly associated with IRD include Mycobacterium avium, Mycobacterium tuberculosis, Cryptococcus neoformans, cytomegalovirus (CMV), Hepatitis C virus (HCV), Hepatitis B virus (HBV) and Varicella Zoster virus (VZV). Risk factors for IRD include CD4+ T-cell counts below 50 cells $/ \mu \mathrm{L}$ prior to ART and an active or sub-clinical infection by an opportunistic pathogen [2]. Chemokines are implicated in IRD. Plasma levels of CXCL10, soluble (s) CD30 (a marker of T-cell activation), CCL2 and IL-18 (a monokine required for T-cell activation) were all correlated with alaninetransaminase levels in $36 \mathrm{HBV}$ coinfected patients after 8 weeks on ART [13]. Increased plasma levels of CXCL10 were associated with TBIRD in a cohort of 75 patients from Cambodia. When considered together, high CXCL10, low CCL2 and high IL-18 levels before ART were significant predictors of TB-IRD [9].

HIV and ART can cause peripheral SN [1]. Both SN triggered by antiretroviral drugs [NRTI-SN] and HIVinduced Distal Sensory Polyneuropathy (DSP) [14] are characterised by pain and/or numbness in the feet, typically with reduced ankle reflexes and/or sensory signs [15]. DSP involves disordered inflammation, with activated macrophages and cytokines (including $\mathrm{TNF} \alpha$ ) observed around peripheral nerves in affected patients [16,17] and inflammatory genotypes are implicated in NRTI-SN [18]. There is also in vivo and in vitro evidence that aberrant host inflammatory responses underpin painful neuropathies in other settings [4].

This study addresses two questions. How does ART affect circulating plasma chemokines and are these changes reflected in the plasma of HIV patients with
ART-associated inflammatory diseases. The study is based on a cohort of patients initiating ART in Malaysia with advanced immunodeficiency and many opportunistic infections.

\section{Materials and methods}

\subsection{Study subjects}

Antiretroviral-naive HIV-infected patients attending outpatient clinics at the University of Malaya Medical Centre (Kuala Lumpur, Malaysia) were recruited for a longitudinal study. All patients $(n=66)$ began ART with CD4+ T-cell counts $<200 / \mu$ l and donated plasma at baseline, 6, 12, 24 and 48 weeks. Where all data were available $(n=56)$, all patients achieved a $>2$-fold recovery of CD4+ T-cell count and/or CD4+ T-cells $>200 / \mu$ l during ART. 28 healthy control subjects from Kuala Lumpur donated blood once. DNA was collected from peripheral blood neutrophils from all participants. Laboratory, clinical and demographic data were collected from patients' medical records. The study was approved by the Ethics Committees of the University of Malaya and informed consent was obtained from all participants.

Fifteen patients experienced at least one IRD event, including tuberculosis (TB; $n=7$ ), Kaposi's sarcoma $(n=3), \mathrm{CMV}(n=3)$, dermatomal VZV $(n=1)$ and cryptococcal meningitis $(n=1)$. IRD cases were analysed together as the numbers are low. No coinfection with HBV or HCV was reported.

Thirty patients were screened for neuropathy using the AIDS Clinical Trials Group Brief Peripheral Neuropathy Screening Tool (ACTG BPNS) [19] at least 6 months after commencing ART. SN was defined by the presence of both symptoms and signs on the ACTG BPNS.

\subsection{Sample collection and CD4+ T-cell counts}

EDTA-treated blood was collected from patients at baseline and weeks 6, 12, 24 and 48. Peripheral blood mononuclear cells were isolated by Ficoll gradient centrifugation and plasma and neutrophils were stored at $-80^{\circ} \mathrm{C}$. CD4+ T-cell counts were measured in EDTAtreated whole blood by routine flow cytometry.

\subsection{Quantification of chemokines in plasma}

Plasma CCL5 was quantitated using DuoSet ELISA (R\&D systems, Minneapolis) and CCL2 and CXCL10 
were quantitated using BDOptEIA ELISA (BDBiosciences). Absorbance was read at 450nm and Microplate Manager software (version 5.2; Bio-Rad) was used for analysis. The lowest limits of detection for the CCL5, CCL2 and CXCL10 assays were 15, 1 and $5 \mathrm{pg} / \mathrm{mL}$, respectively.

\subsection{DNA extraction and SNP genotyping}

DNA was extracted from blood neutrophils using a QIAamp DNA Blood Mini Kit (QIAGEN; Valencia, $\mathrm{CA}$ ) and stored at $-80^{\circ} \mathrm{C}$. Eleven SNP were genotyped using Taqman FAM or VIC-labelled probes [C__1587 4407_10; rs2107538 (CCL5), C__15944115_20; rs2069 705 (IFN $\gamma$ ), C__27478341_10; rs3760396 (CCL2), C_15874396_20; rs2280788 (CCL5), C__11939405_1_; rs4586 (CCL2), C__-7449817_10; rs1024610 (CCL2), C___1085600_10; rs1799945 (HFE), C_-_9546517_10; rs1143634 (ILIB), C_-_1839943_10; rs16944 (ILIB), C___2590362_10; rs1024611 (CCL2), C__1085595_10; rs1800562 (HFE)] and Universal PCR Master Mix (Applied Biosystems; Foster City, CA). Aliquots of 10ng were dried down on 384-well microtiter plates and amplified on a Roche LC480 (Roche Applied Science; Indianapolis, IN). Thermocycling used one step of 10 min at $95^{\circ} \mathrm{C}$ followed by 40 cycles of denaturation at $92^{\circ} \mathrm{C}$ for $15 \mathrm{~s}$ with annealing and extension at $60^{\circ} \mathrm{C}$ for $1 \mathrm{~min}$. Fluorescence was read on a Roche LC480 using proprietary allelic discrimination software. Repeats and non-template controls were included in each run. The median (range) genotyping success rates were 99 (98-100) \% for each SNP in all cohorts.

\section{Statistical analysis}

Plasma chemokine data were assessed using the Graphpad Software Package (Graphpad Software Inc; San Diego, CA) and Stata version 10.1 (StataCorp, College Station, Texas, USA). Demographic details, plasma chemokine levels and minor allele carriage were compared between groups using $\mathrm{Chi}^{2}$ or Fisher's Exact tests (dichotomous variables), Wilcoxon ranksum or Mann-Whitney paired tests [non-normally distributed continuous variables]. Correlations between chemokine levels and time on ART were assessed using linear regression modelling, clustered by patient to account for multiple measures from each individual. Chemokine levels were log transformed to approximate normality. Correlations between chemokine levels and CD4+ T-cell counts were analysed using Spear- man's test, [non-normally distributed continues variables]. Allele and genotype frequencies in each ethnic group conformed with Hardy-Weinberg equilibrium (HWE) when assessed using GENEPOP v3.3 [20].

\section{Results}

\subsection{Time on ART influences chemokine levels in a longitudinal cohort}

The effects of ART on plasma chemokine levels are shown in Fig. 1. CXCL10 levels were lower after 6 weeks on ART compared to baseline ( $p=0.002-$ $<0.0001$; Wilcoxon rank-sum), but remained higher than healthy controls ( $p \leqslant 0.0001$; Mann Whitney). The association between decreasing log CXCL10 levels and time on ART was confirmed using a linear regression model clustered by patient $\left(p \leqslant 0.0001, r^{2}=\right.$ 0.18) (Fig. 1a).

Plasma CCL5 levels rose slowly during ART and were higher than baseline from week 6 to week 48 ( $p=$ 0.04-0.006; Wilcoxon rank-sum). However, plasma levels of CCL5 measured in HIV patients (either at baseline or during ART) were similar to healthy controls ( $p \geqslant 0.1$; Mann Whitney) (Fig. 1b).

Levels of CCL2 were below the limit of detection in most samples, so we saw no clear trends over time. However, CCL2 was detectable in 101 of 324 (31\%) of samples from HIV patients, compared with only 5 of $38(13 \%)$ from controls ( $p=0.02$; $\mathrm{Chi}^{2}$ test). CCL2 levels were higher in HIV patients than controls at all time points from week 12 ( $p=0.04-p=0.01$; Mann Whitney) (Fig. 1c).

A rise in CD4+ T-cell counts with time on ART was evident in the entire cohort as expected (Fig. 1d). We found no associations between CD4+ T-cell recovery and plasma levels of any chemokine measured here ( $p=0.63-0.92$; Mann Whitney). Baseline CD4+ Tcell counts were also not predictive of chemokine levels ( $r=-0.007-0.17, p=0.1-0.8$; Spearmen's test $)$.

\subsection{Plasma levels of CXCL1O and CCL5 are elevated in IRD patients}

Plasma CXCL10 levels fell by week 6 compared to baseline levels in patients with no IRD ( $p=0.001$; Wilcoxon-rank sum), but did not decline significantly in IRD patients ( $p=0.09-0.8$ ) (Fig. 2a).

Plasma CCL5 concentrations were similar to control donors in patients without IRD $(p \geqslant 0.2$ at all time 


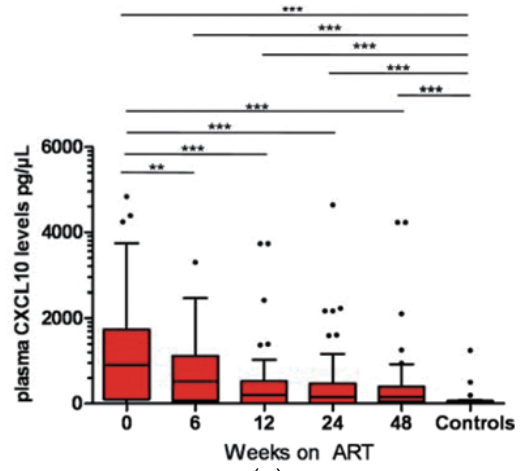

(a)

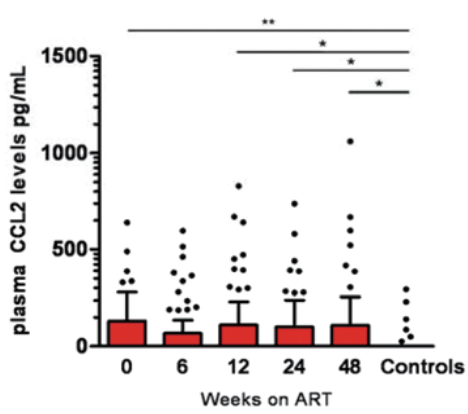

(c)

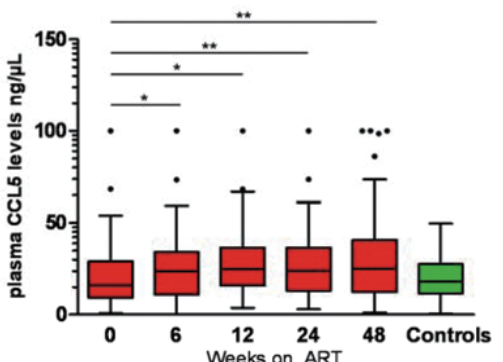

(b)

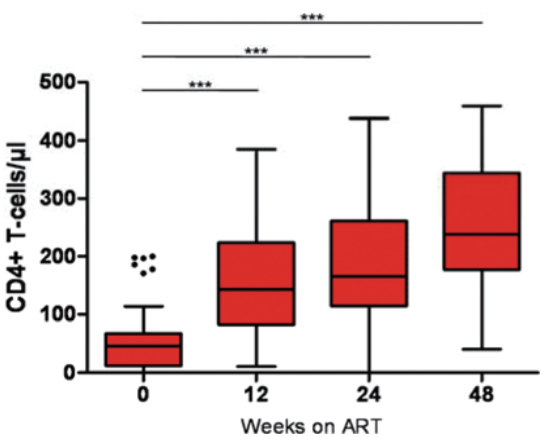

(d)

Fig. 1. Chemokine levels and CD4+ T-cell counts over time in HIV patients initiating ART. The $x$ axis shows number of weeks on ART. The y axis indicates plasma concentrations of (a) CXCL10, (b) CCL5 and (c) CCL2, and (d) CD4+ T-cell $\operatorname{count}^{*} p \leqslant 0.05 ;{ }^{* *} p \leqslant 0.01 ;{ }^{* * *} p \leqslant$ 0.001 .

points tested; Mann Whitney) but higher than controls in IRD patients. This difference was significant at weeks 6, 12 and 48 ( $p=0.03, p=0.0004, p=0.003$ respectively) (Fig. 2b).

\subsection{Plasma levels of CCL5 are high in some patients with $S N$}

This study had limited power to explore associations between HIV-SN and plasma chemokine levels, with only 30 patients screened for SN. However three of 8 patients with SN displayed persistently elevated plasma levels of CCL5 on ART (Fig. 3). This was not apparent in any of the 22 patients without $\mathrm{SN}$ ( $p=0.01$; Fisher's test). The finding warrants further consideration in larger cohorts where SN status is followed at baseline as well as during ART. Patterns of plasma CCL2 and CXCL10 over time were similar in HIV patients with and without $\mathrm{SN}$.

\subsection{Chemokine Genotyping}

A panel of SNPs in the CCL5, CCL2 and IFN $\gamma$ genes was chosen based on reported associations with plas- ma chemokine levels or inflammatory diseases. Allele and genotype frequencies in each ethnic group conformed with Hardy-Weinberg equilibrium (HWE) when assessed using GENEPOP v3.3 [20]. No associations were observed between SNPs and CD4+ T cell recovery, the development of SN or an IRD, or plasma chemokine levels - other than a modest association between increased baseline plasma CXCL10 and carriage of rs 1024610 minor allele ( $p=0.05$; Fisher's test). This association may be random as the SNP is in CCL2. No association was observed between baseline CD4+ T cell counts and any of the tested SNPs ( $p=$ $0.06-0.8$; Fisher's test)

\section{Discussion}

This study assessed changes in plasma chemokine levels in a longitudinal study of patients beginning ART with advanced HIV disease. CXCL10 levels fell on ART, consistent with our findings in a Cambodian cohort [9]. However CXCL10 levels remain higher in Malaysian HIV patients at all time points compared to 


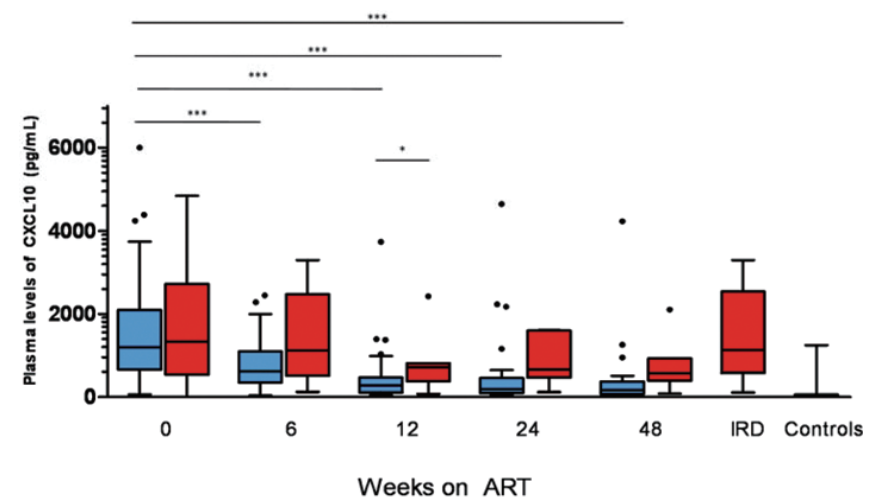

(a)

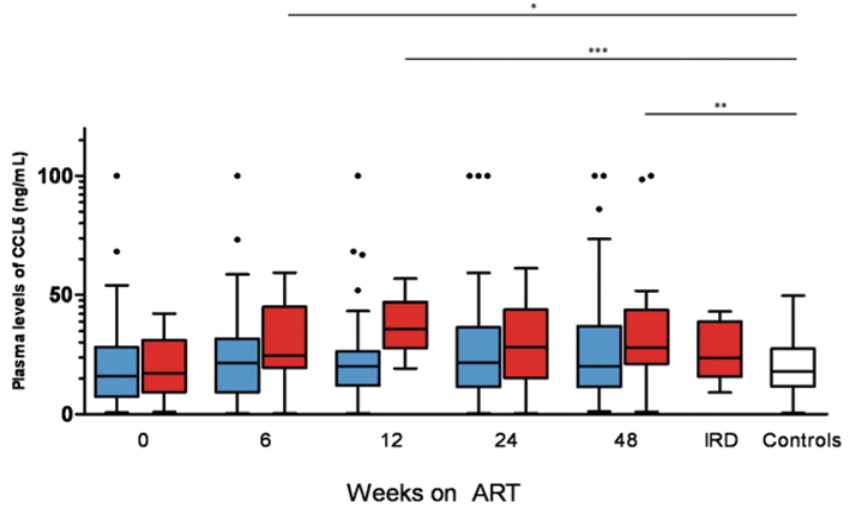

(b)

Fig. 2. Chemokine levels over time in patients initiating ART in patients who did (red) and did not (blue) develop an IRD. The $\mathrm{x}$ axis indicates weeks on ART. The y axis indicates plasma concentrations of (a) CXCL10 and (b) CXCL5. ${ }^{*} p \leqslant 0.05 ;{ }^{* *} p \leqslant 0.01 ;{ }^{* * *} p \leqslant 0.001$.

healthy controls. Importantly CXCL10 levels did not decline to the same extent in IRD patients despite 48 weeks of effective ART. This has been described in TB IRD [9] and HBV IRD [13]. CXCL10 is induced by IFN $\gamma$, and is responsible for recruiting T-cells to sites of inflammation and activation of NK cells. The persistently elevated levels of CXCL10 observed in patients with IRD may contribute to the inflammation characteristic of IRD.

In contrast to CXCL10, CCL5 levels rose as patients responded to ART but did not differ significantly from healthy controls. It is also notable that the increase in CCL5 plasma levels during ART is less than the decrease of CXCL10 levels in patients on ART, indicating that CXCL10 is possibly a more useful monitor of patient response to ART. Elevated plasma CCL5 levels were seen in 3 out of 8 patients who experienced SN, but not in ART-treated patients without SN. It is plausible that elevated CCL5 may contribute to the development of SN in some patients, with in vitro studies in cultured dorsal root ganglia (DRG) finding CCL5-CCR5 ligation created neurotoxicity similar to that induced by gp-120 [21]. Moreover administration of CCL5 to adult rat hind paw produced dose-dependent tactile allodynia [22]. CCL5 can promote production of the pro-inflammatory cytokine $\mathrm{TNF} \alpha$ in co-culture [23], possibly leading to TNFR1-mediated neurotoxicity and resulting neuropathy.

Plasma CCL2 was frequently below the limit of detection for our assay. Nonetheless, detectable CCL2 was more common in patients than controls, so this chemokine warrants further investigation. Detectable levels of plasma CCL2 were not associated with IRD or SN. CCL2 inhibits viral entry in peripheral blood lymphocytes via competitive binding of the CCR2 and CCR5 receptors $[24,25]$. CCL2 is produced in response to pro-inflammatory stimuli and attracts monocytes, macrophages, basophils, mast cells, T lymphocytes, natural killer cells and dendritic cells to sites of injury [26]. This range of effects illustrates the complexity that needs to be considered in future studies. 

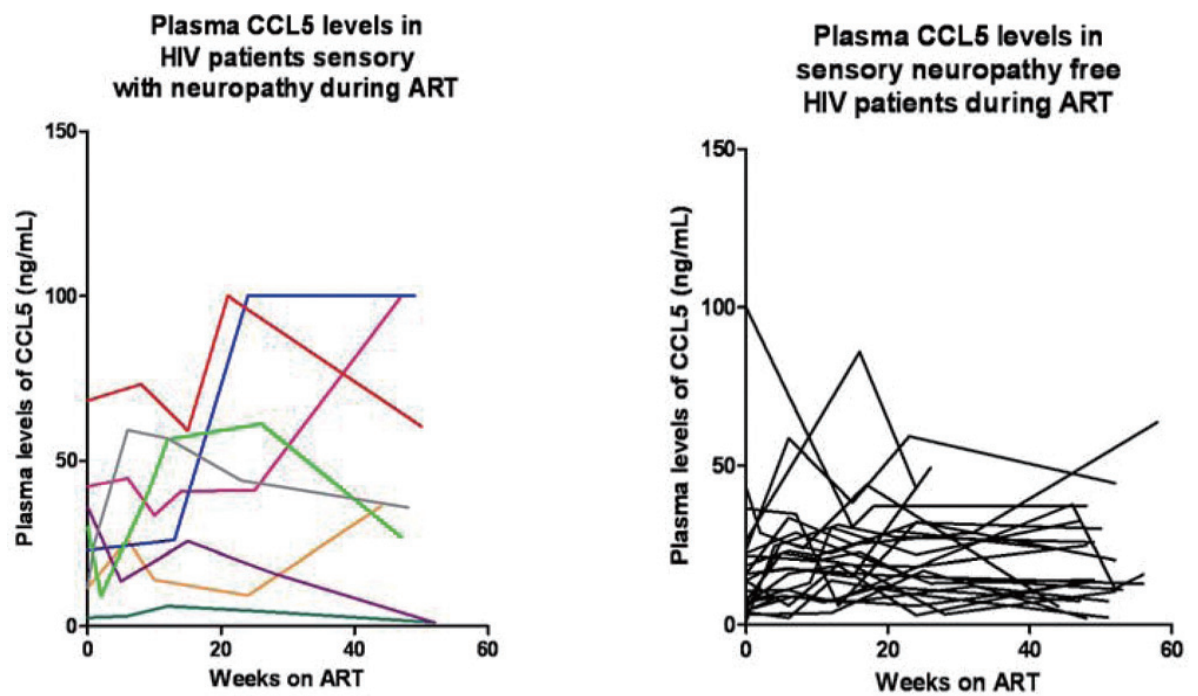

Fig. 3. Plasma concentrations of CCL5 in (a) individual patients with sensory neuropathy and (b) patients without neuropathy.

No genotypes tested affected chemokine levels or disease status. The panel of SNPs were selected from published papers [27-34]. All were associated with inflammatory disorders or with a change in chemokine levels in vivo or in vitro. Several IRD have been associated with low baseline CD4+ T-cell counts (summarized in [35]) and with steady high levels of CXCL10 on ART $[9,13]$. Hence we examined whether high levels of CXCL10 were a feature of low baseline counts. However we found no association between CXCL10 levels and CD4+ T-cell counts at any time before or during treatment.

There were several limitations to our study. Firstly, as this was a preliminary study, the cohort was small, patients with any IRD were assessed together - using the hypothesis that all IRD represent an immunopathology [36]. However different mechanisms may mediate IRD associated with different pathogens [3], so future studies should assess these separately. Similarly the study was not adequately powered to explore associations between chemokine levels and SN, highlighting the need for a larger cohort. Future studies should also include assessments of immune activation and assessment of SN status prior to ART (to assist in distinguishing DSP from cases of SN developing on ART).

In summary; plasma levels of CXCL10 decreased and CCL5 increased during ART. CCL2 levels were elevated by HIV disease, but did not change on ART. Patients reporting an IRD maintained high levels of CXCL10 compared to non IRD patients, Some patients with SN displayed elevated levels of CCL5 on ART indicating that altered chemokines levels are possibly one of a multitude of factors contributing to aetiology of this condition. Chemokine levels were not associated with CD4+ T-cell counts before and on ART. These data suggest that chemokines play a role in the inflammatory pathways of IRD and SN, but do not support a role for the chemokines studied in CD4+ T-cell recovery on ART.

\section{References}

[1] C.L. Cherry et al., Nucleoside analogues and neuropathy in the era of HAART, J Clin Virol 26(2) (2003), 195-207.

[2] M.A. French, P. Price and S.F. Stone, Immune restoration disease after antiretroviral therapy, AIDS 18(12) (2004), 16151627.

[3] P. Price et al., Immune restoration diseases reflect diverse immunopathological mechanisms, Clin Microbiol Rev 22(4) (2009), 651-663.

[4] N. Uceyler et al., Differential expression of cytokines in painful and painless neuropathies, Neurology 69(1) (2007), 42-49.

[5] L. Kestens, N. Seddiki and P.R. Bohjanen, Immunopathogenesis of immune reconstitution disease in HIV patients responding to antiretroviral therapy, Curr Opin HIV AIDS 3(4) (2008), 419-424.

[6] P. Aukrust, F. Muller and S.S. Froland, Circulating levels of RANTES in human immunodeficiency virus type 1 infection: effect of potent antiretroviral therapy, J Infect Dis 177(4) (1998), 1091-1096.

[7] L.R. Bisset et al., Change in circulating levels of the chemokines macrophage inflammatory proteins 1 alpha and 11 beta, RANTES, monocyte chemotactic protein-1 and interleukin-16 following treatment of severely immunodeficient HIV-infected individuals with indinavir, AIDS 11(4) (1997), 485-491.

[8] S. Fransen et al., RANTES production by T cells and CD8mediated inhibition of human immunodeficiency virus gene 
expression before initiation of potent antiretroviral therapy predict sustained suppression of viral replication, J Infect Dis 181(2) (2000), 505-512.

[9] B.G.E. Oliver, J.H. Price, P. Phillips, M. Saphonn and V. Chhi Vun, Mediators of innate and adaptive immune responses differentially affect immune restoration disease associated with Mycobacterium tuberculosis in HIV patients beginning ART, J Infect Dis 202(11) (2010), 1728-1737.

[10] K.I. Relucio et al., Proteomic analysis of serum cytokine levels in response to highly active antiretroviral therapy (HAART), J Proteome Res 4(2) (2005), 227-231.

[11] E. Stylianou et al., Interferons and interferon (IFN)-inducible protein 10 during highly active anti-retroviral therapy (HAART)-possible immunosuppressive role of IFN-alpha in HIV infection, Clin Exp Immunol 119(3) (2000), 479-485.

[12] S.F. Stone, P. Price and M.A. French, Immune restoration disease: a consequence of dysregulated immune responses after HAART, Curr HIV Res 2(3) (2004), 235-242.

[13] M. Crane et al., Immunopathogenesis of hepatic flare in HIV/ hepatitis B virus (HBV)-coinfected individuals after the initiation of HBV-active antiretroviral therapy, J Infect Dis 199(7) (2009), 974-981.

[14] S.C. Keswani et al., HIV-associated sensory neuropathies, Aids 16(16) (2002), 2105-2117.

[15] Y.T. So et al., Peripheral neuropathy associated with acquired immunodeficiency syndrome. Prevalence and clinical features from a population-based survey, Arch Neurol 45(9) (1988), 945-948.

[16] I. Nagano et al., Increased NADPH-diaphorase reactivity and cytokine expression in dorsal root ganglia in acquired immunodeficiency syndrome, J Neurol Sci 136(1-2) (1996), 117128.

[17] S.L. Wesselingh et al., Cytokine dysregulation in HIV-associated neurological disease, Adv Neuroimmunol 4(3) (1994), 199-206.

[18] C.L. Cherry et al., Cytokine genotype suggests a role for inflammation in nucleoside analog-associated sensory neuropathy (NRTI-SN) and predicts an individual's NRTI-SN risk, AIDS Res Hum Retroviruses 24(2) (2008), 117-123.

[19] C.L. Cherry et al., Evaluation of a clinical screening tool for HIV-associated sensory neuropathies, Neurology $\mathbf{6 5}(11)$ (2005), 1778-1781.

[20] F. Rousset and M. Raymond, Testing heterozygote excess and deficiency, Genetics 140(4) (1995), 1413-1419.

[21] S.C. Keswani et al., Schwann cell chemokine receptors mediate HIV-1 gp120 toxicity to sensory neurons, Ann Neurol 54(3) (2003), 287-296.

[22] S.B. Oh et al., Chemokines and glycoprotein 120 produce pain hypersensitivity by directly exciting primary nociceptive neurons, J Neurosci 21(14) (2001), 5027-5035.
[23] L. Qiu et al., Induction of copper/zinc-superoxide dismutase by CCL5/CCR5 activation causes tumour necrosis factoralpha and reactive oxygen species production in macrophages, Immunology 128(1 Suppl) (2009), e325-e334.

[24] J.M. Frade et al., The amino-terminal domain of the CCR2 chemokine receptor acts as coreceptor for HIV-1 infection, $J$ Clin Invest 100(3) (1997), 497-502.

[25] J.W. Homan et al., Inhibition of morphine-potentiated HIV1 replication in peripheral blood mononuclear cells with the nuclease-resistant 2-5A agonist analog, 2-5A(N6B), J Acquir Immune Defic Syndr 30(1) (2002), 9-20.

[26] L. Gu et al., In vivo properties of monocyte chemoattractant protein-1, J Leukoc Biol 62(5) (1997), 577-580.

[27] A. Zhernakova et al., Genetic variants of RANTES are associated with serum RANTES level and protection for type 1 diabetes, Genes Immun 7(7) (2006), 544-549.

[28] M. Mezger et al., Polymorphisms in the chemokine (C-X-C motif) ligand 10 are associated with invasive aspergillosis after allogeneic stem-cell transplantation and influence CXCL10 expression in monocyte-derived dendritic cells, Blood 111(2) (2008), 534-536.

[29] C. Alonso-Villaverde et al., Host-pathogen interactions in the development of metabolic disturbances and atherosclerosis in HIV infection: the role of CCL2 genetic variants, Cytokine 51(3), 251-258.

[30] W.S. Modi et al., MCP-1-MCP-3-Eotaxin gene cluster influences HIV-1 transmission, AIDS 17(16) (2003), 2357-2365.

[31] D.H. McDermott et al., CCL2 polymorphisms are associated with serum monocyte chemoattractant protein-1 levels and myocardial infarction in the Framingham Heart Study, Circulation 112(8) (2005), 1113-1120.

[32] A.R. Kallianpur et al., Hemochromatosis (HFE) gene mutations and peripheral neuropathy during antiretroviral therapy, AIDS 20(11) (2006), 1503-1513.

[33] N. Buchs et al., IL-1B and IL-1Ra gene polymorphisms and disease severity in rheumatoid arthritis: interaction with their plasma levels, Genes Immun 2(4) (2001), 222-228.

[34] J.K. Yamamoto-Furusho et al., Interleukin 1 beta (IL-1B) and IL-1 Antagonist Receptor (IL-1RN) Gene Polymorphisms are Associated With the Genetic Susceptibility and Steroid Dependence in Patients With Ulcerative Colitis, J Clin Gastroenterol.

[35] M. Muller et al., Immune reconstitution inflammatory syndrome in patients starting antiretroviral therapy for HIV infection: a systematic review and meta-analysis, Lancet Infect Dis 10(4), 251-261.

[36] I. Sereti, A.J. Rodger and M.A. French, Biomarkers in immune reconstitution inflammatory syndrome: signals from pathogenesis, Curr Opin HIV AIDS 5(6), 504-510. 


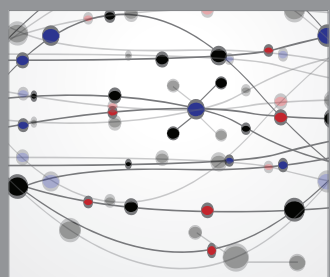

The Scientific World Journal
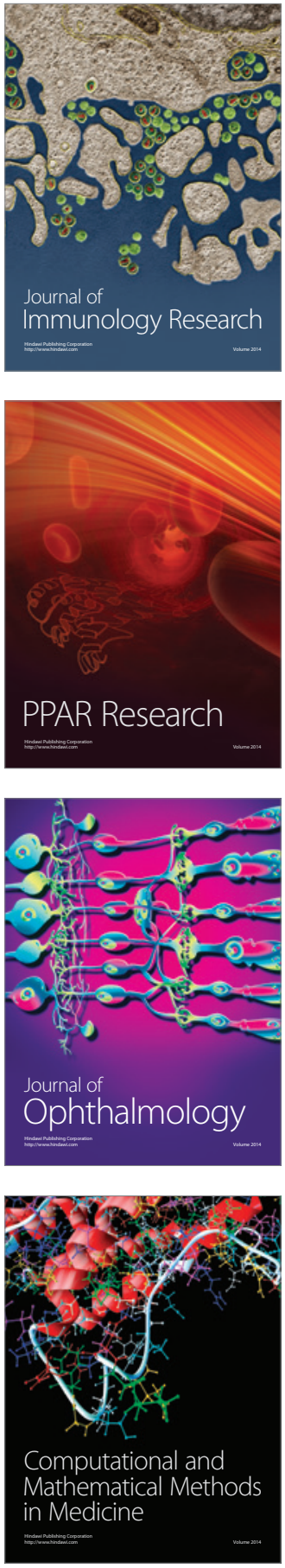

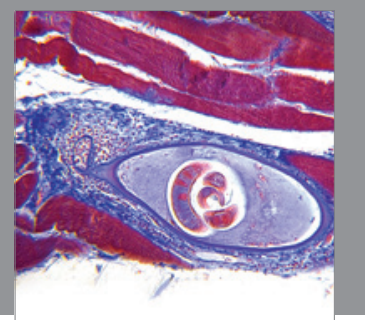

Gastroenterology

Research and Practice
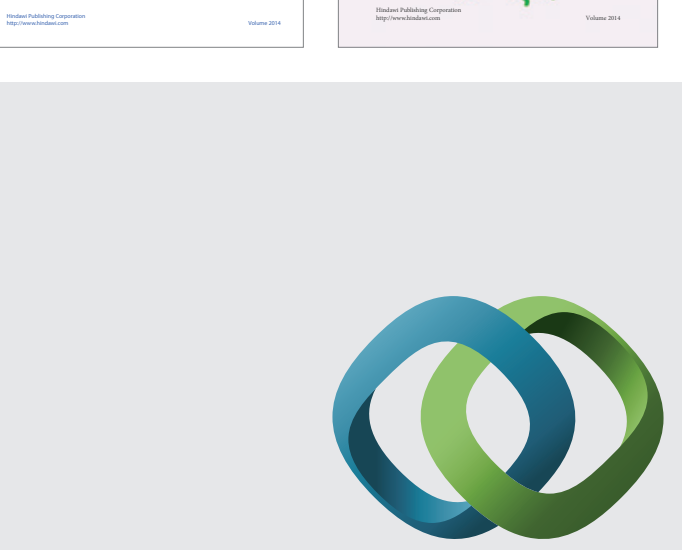

\section{Hindawi}

Submit your manuscripts at

http://www.hindawi.com
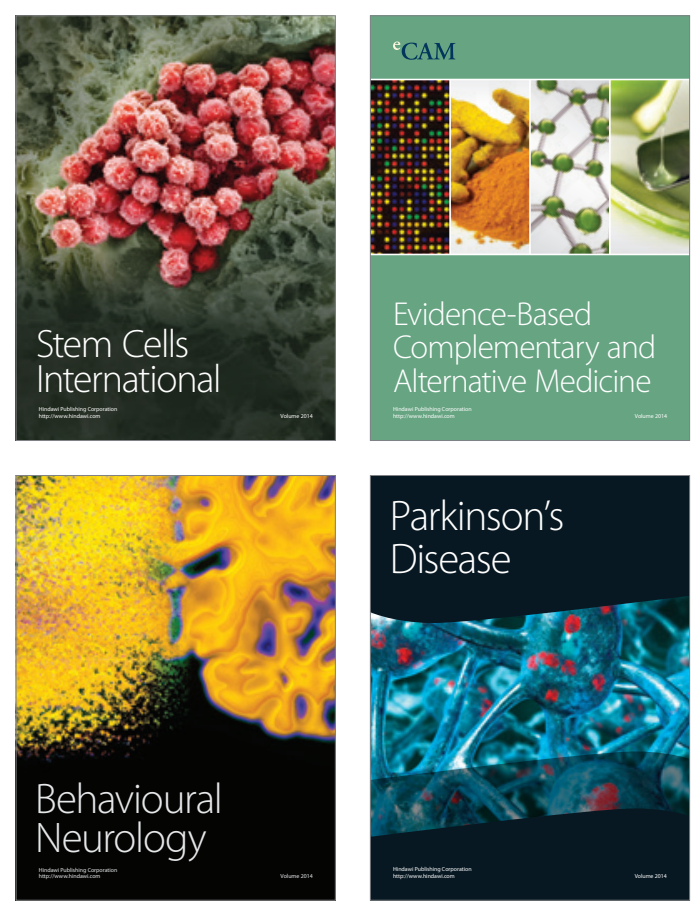

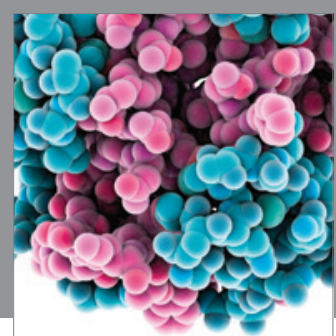

Journal of
Diabetes Research

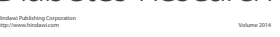

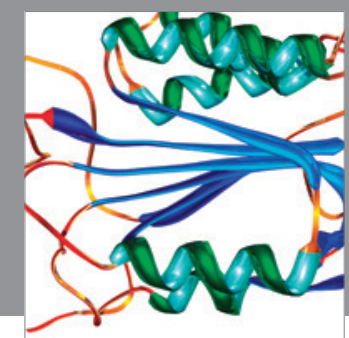

Disease Markers
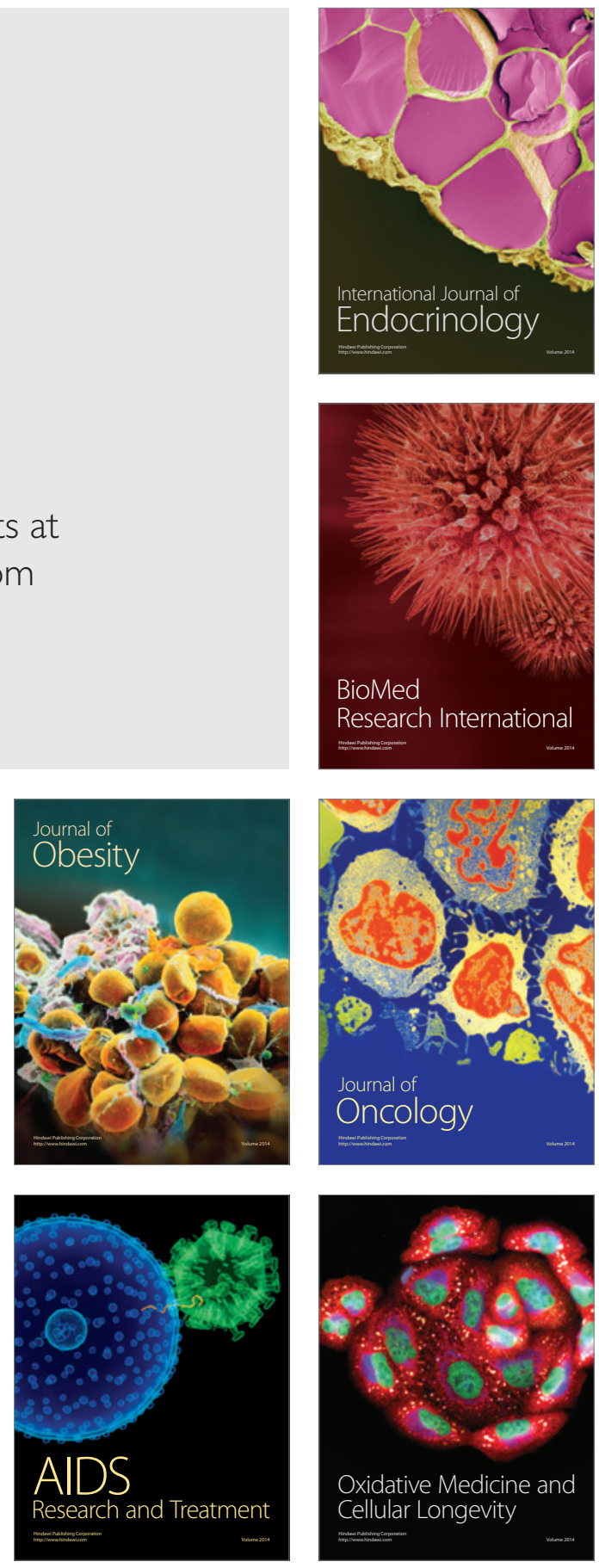